03;05;12

\title{
Влияние индуктивности электрической цепи на энергетические характеристики разряда в воде и деформирования \\ пластин
}

\section{(C) В.М. Косенков, В.М. Бычков}

Институт импульсных процессов и технологий НАН Украины, Николаев, Украина

E-mail:v.m.kosenkov@gmail.com

Поступило в Редакцию 27 января 2017 г.

Выполнено экспериментальное исследование влияния индуктивности разрядной цепи на эффективность преобразования энергии, запасаемой в конденсаторной батарее, в канале электрического разряда в воде и в процессе пластического деформирования пластин. Впервые определено положительное влияние увеличения индуктивности разрядной цепи на деформирование пластин, приводящее к увеличению количества используемой для этого энергии.

DOI: 10.21883/PJTF.2017.16.44939.16706

Высоковольтный разряд в воде находит широкое применение в различных технических приложениях [1-4]. Его эффективность во многом зависит от параметров разрядной цепи, таких как емкость конденсаторной батареи $(C)$, напряжение ее заряда $\left(U_{0}\right)$, индуктивность $(L)$, расстояние между электродами $\left(l_{c}\right)$, активное сопротивление $\left(R_{r}\right)$ всех элементов разрядной цепи, кроме канала разряда. Во многих работах [2-9] исследовалось влияние параметров $C, U_{0}, l_{c}, R_{r}$ на изменение свойств различных сред и объектов под действием волн давления, генерируемых электрическим разрядом в воде, но влиянию $L$ уделялось намного меньше внимания. Главным образом это связано с тем, что в большинстве технических приложений основное воздействие на среды и объекты осуществляют первичные ударные волны, генерируемые электрическим разрядом в воде [1,2]. Положительный эффект воздействия ударных волн на среды и объекты, как правило, пропорционален их амплитуде. Было определено [5], что уменьшение $L$ приводит к существенному увеличению амплитуды первичных ударных волн. Поэтому во многих 


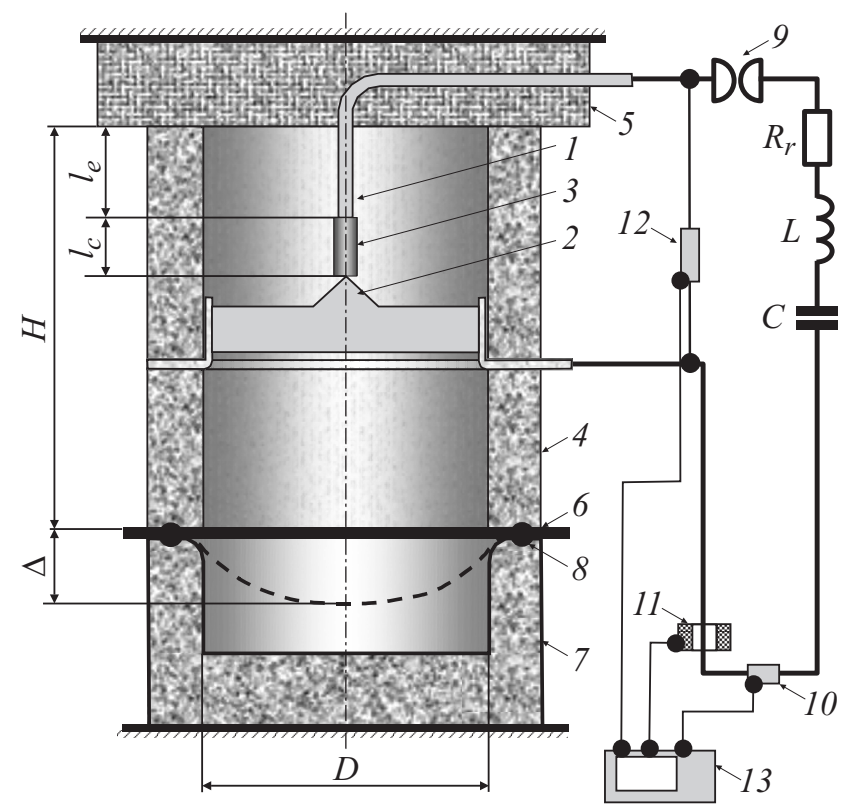

Рис. 1. Схема эксперимента.

работах $[1,5]$ рекомендовалось задавать $L$ как можно меньшей величины. По аналогии эти рекомендации распространялись на процессы, время протекания которых намного больше, чем время действия первичных ударных волн. К таким процессам относится деформирование пластин, происходящее в результате электрического разряда в воде. В этом случае данные о влиянии величины $L$ отсутствуют, что определяет актуальность проведения исследований.

Цель настоящей работы заключалась в экспериментальном исследовании влияния индуктивности разрядной цепи на количество энергии, выделяемой в канале разряда, а также на энергию деформирования металлических пластин под действием волн, генерируемых электрическим разрядом в воде.

В процессе исследований использовалось экспериментальное оборудование, схема которого представлена на рис. 1. Разряд осуществлялся

Письма в ЖТФ, 2017, том 43, вып. 16 
между двумя электродами 1 и 2 , установленными на расстоянии $l_{c}$ друг от друга, между которыми в результате пробоя слоя воды формировался канал разряда 3. Электродная система 1 и 2 устанавливалась в разрядной камере 4, состоящей из двух толстостенных цилиндров с общей высотой $H=340 \mathrm{~mm}$ и внутренним диаметром $D=120 \mathrm{~mm}$, между которыми размещался электрод 2. Сверху разрядная камера 4 закрывалась пластиной стеклотекстолита 5, которая также служила высоковольтной изоляцией электрода 1 , выступающего за ее пределы на $l_{e}(70 \mathrm{~mm})$. Снизу разрядная камера 4 ограничена пластиной 6 из высокопрочного алюминиевого сплава 5754 толщиной $1.35 \mathrm{~mm}$, установленной на цилиндрической матрице 7 и прижатой корпусом разрядной камеры 4. От перемещения в радиальном направлении из фланцев пластина 6 фиксировалась стопорным кольцом 8. Полость разрядной камеры 4 между пластинами 5 и 6 заполнялась водой.

Электроды 1 и 2 подключались к разрядной цепи, схема замещения которой характеризуется параметрами $C, L$ и $R_{r}$ (рис. 1). Для коммутирования разрядной цепи использовался воздушный разрядник 9 , в результате замыкания которого между электродами 1 и 2 возникала разность потенциалов $U_{0}$, приводящая к формированию между ними канала разряда 3. Канал разряда 3 в процессе расширения генерировал волны давления, распространяющиеся в воде и приводящие к деформированию пластины 6 до максимального прогиба $\Delta$. Форма прогиба пластины 6 показана на рис. 1 штриховой линией. Электрод 2 выполнен в виде пластины, расположенной в диаметральной плоскости разрядной камеры 4, и поэтому практически не влиял на распространение волн в осевом и радиальном направлениях.

Во время экспериментов емкость конденсаторной батареи $C$ была постоянной и равной $10 \mu \mathrm{F}$. Индуктивность $L$ и активное сопротивление $R_{r}$ всех элементов разрядной цепи, кроме канала разряда, определялись по осциллограммам тока короткого замыкания электродов 1 и 2. Минимальная индуктивность разрядного контура равнялась $9.5 \mu \mathrm{H}$, а минимальное активное сопротивление $-0.07 \Omega$. Напряжение заряда конденсаторной батареи $U_{0}$ изменялось в пределах от 30 до $40 \mathrm{kV}$, что соответствовало энергии заряда батареи $\left(E_{0}\right)$ от 4.5 до $8 \mathrm{~kJ}$. Расстояние $l_{c}$ между электродами 1 и 2 задавалось равным 20, 30 и $55 \mathrm{~mm}$. Разряд инициировался пробоем слоя воды между электродами под действием $U_{0}$.

Анализ процессов выполнялся на основе измерения силы тока $(i)$ и напряжения $(U)$ на канале разряда 3 , а также максимального прогиба

Письма в ЖТФ, 2017, том 43, вып. 16 
пластины $6(\Delta)$. Сила тока определялась двумя способами: по падению напряжения на омическом шунте 10 и с помощью пояса Роговского 11. Напряжение на канале разряда измерялось с помощью резистивного делителя напряжения 12. Для записи изменяющихся во времени сигналов использовался цифровой осциллограф 13 марки Tektronix TDS-2024В. Компенсация индуктивной составляющей напряжения на канале разряда осуществлялась по методу, описанному в работе [10].

По результатам измерения силы тока и напряжения на канале разряда определялись энергетические характеристики разряда: мощность $(N)$ и энергия, выделившаяся в канале разряда $\left(E_{c}\right)$. Полученное значение $E_{c}$ делилось на $E_{0}$ для определения эффективности ее передачи в канал разряда $\left(\eta_{c}\right)$. Энергия пластического деформирования пластины $6\left(E_{p}\right)$ находилась по методу [11]. Величина $\eta_{p}$, определяемая как отношение $E_{p}$ к $E_{0}$, характеризовала эффективность использования $E_{0}$ для деформирования пластины 6 .

Изменение $L$ разрядной цепи выполняли, включая в нее последовательно дополнительные катушки с индуктивностью 19.6 и $36 \mu \mathrm{H}$. Активное сопротивление первой из них составляло $0.02 \Omega$, а второй $0.014 \Omega$. Чтобы исключить влияние активного сопротивления катушек на процесс разряда с разной $L$, выполнили серию экспериментов, подключив последовательно в разрядную цепь активные сопротивления, равные сопротивлению катушек. При этом начальная индуктивность разрядной цепи увеличилась до 10.2 и $10 \mu \mathrm{H}$. В результате таких переключений элементов цепи были получены пять соотношений параметров $R_{r}$ и $L: 0.084 \Omega$ и $10 \mu \mathrm{H} ; 0.09 \Omega$ и $10.2 \mu \mathrm{H} ; 0.09 \Omega$ и $29.8 \mu \mathrm{H} ; 0.084 \Omega$ и $46 \mu \mathrm{H} ; 0.104 \Omega$ и $65.6 \mu \mathrm{H}$. Эксперименты с этими соотношениями параметров $R_{r}$ и $L$ выполнялись при одной из трех фиксированных величин $l_{c}: 20,30$ и $55 \mathrm{~mm}$. При каждом соотношении параметров цепи $R_{r}, L$ и $l_{c}$ было выполнено от трех до пяти экспериментов, позволивших произвести статистическую обработку данных и определить погрешность измерений.

Увеличение $L$ от 10 до $65.6 \mu \mathrm{H}$ при одной и той же $E_{0}\left(U_{0}\right.$ равно $30 \mathrm{kV}$ ) увеличивает период колебаний тока в цепи приблизительно в 2.5 раза и во столько же раз уменьшает его амплитуду (рис. 2). Амплитуда силы тока монотонно уменьшается от периода к периоду с течением времени разряда независимо от $L$, при этом амплитуда напряжения между электродами при больших $L$ сначала уменьшается, но к концу разряда увеличивается, достигая уровня напряжения в конце

Письма в ЖТФ, 2017, том 43, вып. 16 


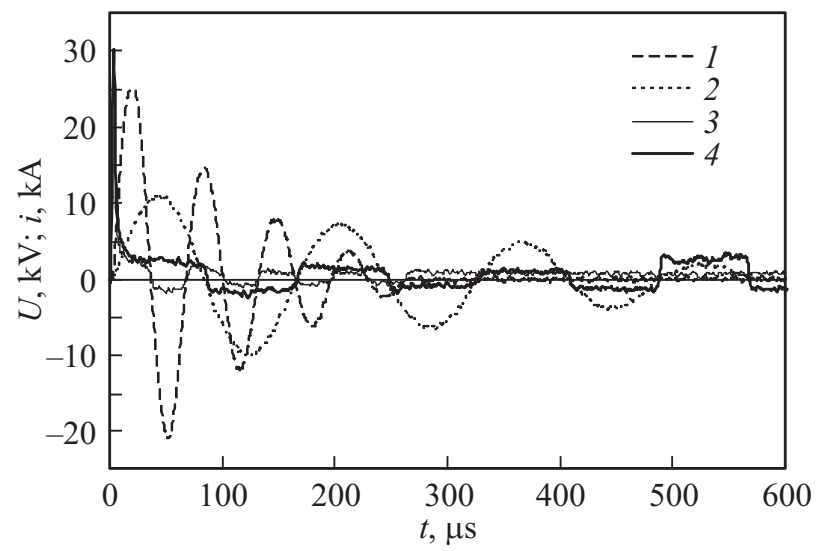

Рис. 2. Электрические характеристики разряда в воде. Цифрами обозначены зависимости от времени: 1 и $2-$ силы тока, 3 и $4-$ напряжения. Линии 1 и 3 получены при $L$, равном $10 \mu \mathrm{H}$, а 2 и $4-65.6 \mu \mathrm{H}$.

первого полупериода разряда. Это свидетельствует о существенном увеличении сопротивления канала разряда на последних периодах колебания тока.

В результате обработки экспериментальных данных получена зависимость $\eta_{c}$ от $L$ (рис. $3, a$ ). Из нее следует, что увеличение $L$ повышает $\eta_{c}$. Степень увеличения $\eta_{c}$ существенно зависит от расстояния между электродами $l_{c}$. Увеличение $L$ приблизительно в 7 раз в случае, когда $l_{c}$ составляет от 20 до $30 \mathrm{~mm}$, приводит к повышению $\eta_{c}$ от 0.53 до 0.7 (в 1.3 раза). Увеличение $l_{c}$ до $55 \mathrm{~mm}$ приводит к меньшему росту $\eta_{c}$ : от 0.65 до 0.72 (в 1.1 раза). Следовательно, увеличение $L$ при малых расстояниях между электродами $l_{c}$ позволяет существенно увеличить $\eta_{c}$.

Для объяснения этого эффекта вычислили эквивалентное сопротивление канала разряда $\left(R_{e}\right)$ по осциллограммам тока и напряжения, используя известную формулу $[5,10]$

$$
R_{e}=\int_{0}^{T_{r}} i(t) U(t) d t / \int_{0}^{T_{r}} i^{2}(t) d t,
$$

где $T_{r}$ — время выделения энергии в канале разряда (в s). 

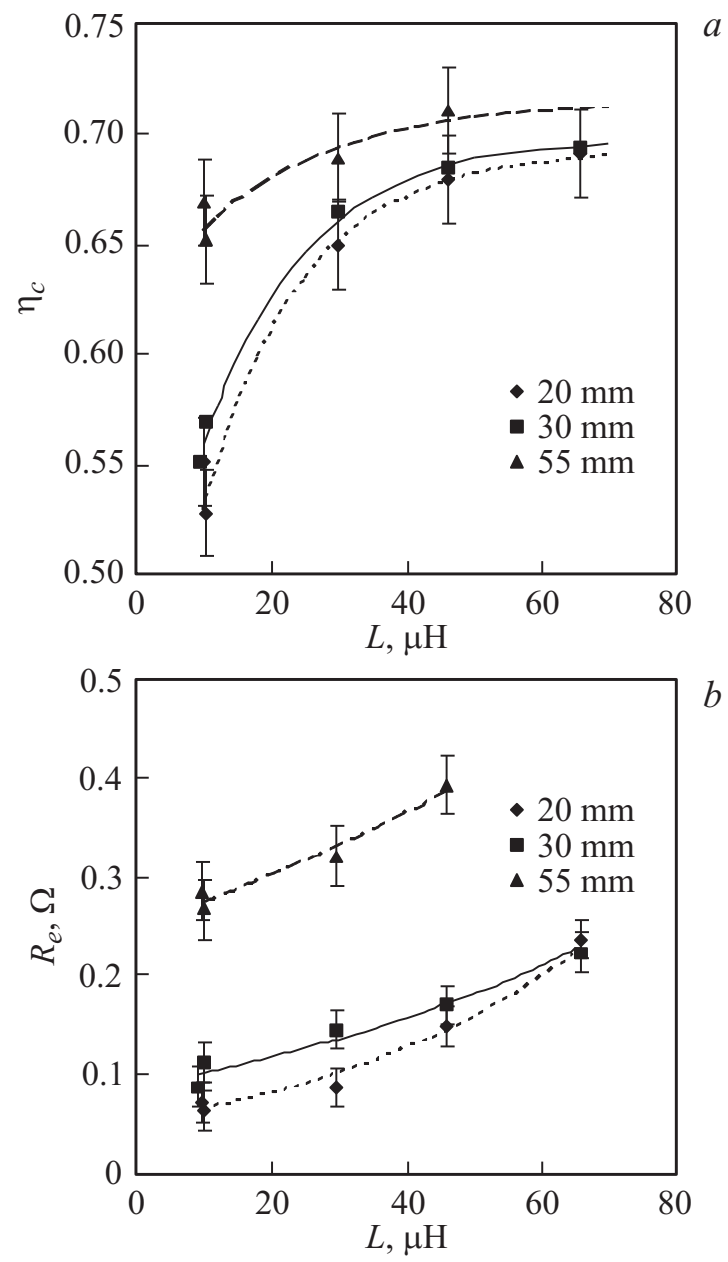

Рис. 3. Влияние индуктивности разрядной цепи на $\eta_{e}(a), R_{e}(b), \eta_{p}(c)$. Точки на частях $a$ и $b$ - экспериментальные данные для различных $l_{c}$, линиями обозначены аппроксимации данных при $l_{c}=20$ (пунктирная), 30 (сплошная) и $55 \mathrm{~mm}$ (штриховая).

Письма в ЖТФ, 2017, том 43, вып. 16 


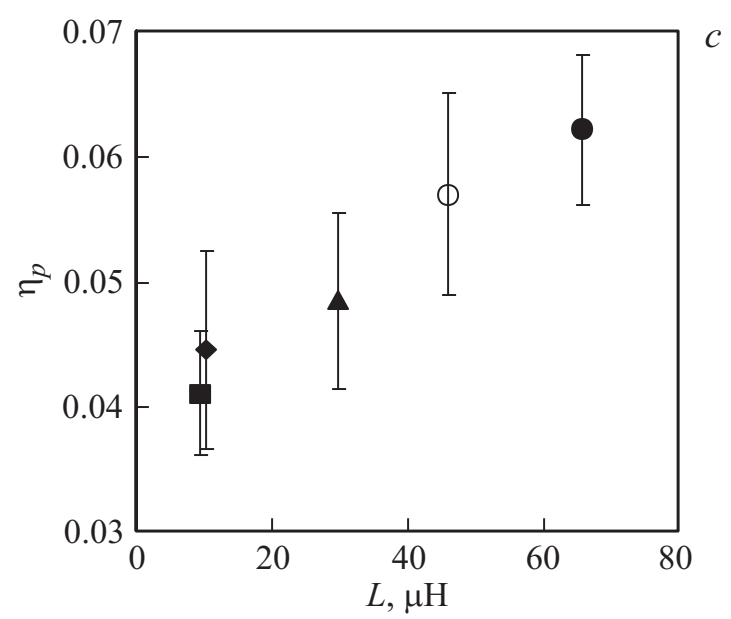

Рис. 3 (продолжение).

Результаты вычислений по формуле (1) показаны на рис. 3, $b$, из которого следует, что увеличение индуктивности разрядного контура существенно увеличивает $R_{e}$. При малых $l_{c}$ величина $R_{e}$ возрастает от 2.5 до 3 раз, а при $l_{c}$, равной $55 \mathrm{~mm}$, - в 1.7 раза. Поскольку $R_{r}$ практически не зависит от изменения $L$, а $R_{e}$ существенно увеличивается, бо́льшая часть энергии $E_{0}$ выделяется в канале разряда, увеличивая тем самым электрический КПД $\left(\eta_{c}\right)$.

Причину возрастания $R_{e}$ в результате увеличения $L$ можно объяснить следующим образом. Увеличение $L$ приводит к большему периоду колебаний тока в разрядном контуре, поэтому максимальная мощность ввода энергии в канал разряда $\left(N_{\max }\right)$ монотонно уменьшается от 2 до 4 раз, а максимальная сила тока $\left(i_{\max }\right)$ - от 2.5 до 3 раз, что уменьшает давление плазмы в канале и, как следствие, скорость его расширения. В результате этого площадь поперечного сечения канала увеличивается медленнее, чем при малой величине $L$, что приводит к росту его электрического сопротивления. Результаты математического моделирования разряда по методу [12] показывают, что увеличение $L$ от 10 до $65.6 \mu \mathrm{H}$ уменьшает плотность тока в канале разряда в 5.5 раза. Это приводит к понижению температуры плазмы в канале и, как следствие, ее электропроводности, что дополнительно увеличивает $R_{e}$.

Письма в ЖТФ, 2017, том 43, вып. 16 
Полученные результаты не противоречат выводам других авторов $[1,5,6]$ о влиянии индуктивности разрядной цепи на силу тока и мощность ввода энергии в канал разряда.

Увеличение $L$ положительно влияет на энергетическую эффективность деформирования пластин. Зависимость $\eta_{p}$ от $L\left(l_{c}=30 \mathrm{~mm}\right)$ показана на рис. 3,c. Она демонстрирует монотонное увеличение $\eta_{p}$ от 0.04 до 0.063 (в 1.6 раза) при увеличении $L$ от 10 до $65.6 \mu \mathrm{H}$. Зависимость $\eta_{p}$ от $L$ можно объяснить влиянием нескольких факторов. Во-первых, увеличивается количество энергии, выделяющейся в канале разряда (рис. 3,a), и, следовательно, больше энергии передается жидкости, действующей на деформируемую пластину, что приводит к увеличению ее деформаций. Во-вторых, более медленный ввод энергии в канал разряда (даже при одинаковой энергии в канале разряда) существенно изменяет процесс обмена энергией и импульсом между ним и деформируемой пластиной. Это приводит к уменьшению амплитуды и градиентов волн давления, генерируемых каналом разряда, но увеличивает длительность их воздействия на деформируемую пластину. При этом уменьшается скорость прогиба пластины, что приводит к понижению интенсивности генерируемых ею волн разрежения и их влияния в результате интерференции с волнами от канала разряда на уменьшение результирующего давления на пластину. В конечном итоге увеличивается суммарный импульс давления жидкости на пластину, что приводит к увеличению ее деформаций.

На основании анализа результатов выполненного экспериментального исследования можно сделать вывод, что в рассмотренной области изменения параметров разрядного контура увеличение $L$ позволяет существенно повысить эффективность преобразования энергии $E_{0}$ в канале разряда до 1.3 раза и в деформируемой пластине до 1.6 раза.

\section{Список литературы}

[1] Гулый Г.А. Научные основы разрядноимпульсных технологий. Киев: Наук. думка, 1990. $311 \mathrm{c.}$

[2] Гаврилов Г.Н. и др. // Разрядноимпульсная технология обработки минеральных сред. Сб. науч. тр. Киев: Наук. думка, 1979. С. 27-31.

[3] Жовноватюк Я.С. // Авиационно-космическая техника и технология. 2010. T. 71. № 4. C. $12-16$.

[4] Hassannejadasla A., Green D.E., Golovashchenko S.F., Samei J., Maris C. // J. Manufactur. Processes. 2014. V. 16. Iss. 3. P. 391-404.

Письма в ЖТФ, 2017, том 43, вып. 16 
[5] Кривищкий E.B. Динамика электровзрыва в жидкости. Киев: Наук. думка, 1986. 208 c.

[6] Наугольных К.А., Рой Н.А. Электрические разряды в воде. М.: Наука, 1977. $155 \mathrm{c}$.

[7] Мамутов А.В., Мамутов В.С. // Научно-технические ведомости СПбГПУ. 2014. T. 190. № 1. C. 101-107.

[8] Косенков В.М., Бычков В.М., Жекул В.Г., Поклонов С.Г. // Письма в ЖТФ. 2012. T. 38. B. 9. C. $103-110$

[9] Косенков В.М. // ЖТФ. 2011. Т. 81. В. 10. С. 133-139.

[10] Щерба А.А., Петриченко С.В. // Техническая электродинамика. 2004. № 3. C. 27-32.

[11] Косенков В.М. // Электронная обработка материалов. 2014. Т. 50. № 2. C. 81-90.

[12] Щерба А.А., Косенков В.М., Бычков В.М. // Электронная обработка материалов. 2015. Т. 51. № 6. С. 71-78. 